Australian Journal of

Educational Technology

\title{
Creating an online teaching space
}

\section{Graeme Hart \\ The University of Melbourne}

\begin{abstract}
In the same way that good classroom teaching uses a variety of techniques to maintain interest and to cater for different student approaches to learning, so too does a good online teaching space require a variety of approaches. A classroom teacher develops teaching strategies both through training and experience. The development of online teaching spaces comes through a knowledge of what the technology can do and experience in how students and teachers react most positively to the technology. This paper reflects on the development of an online web-based teaching site and reports on the stages of development from early beginnings on a local area network to a suggested model online teaching space.
\end{abstract}

\section{Setting the scene - some background information}

Any teaching website needs to be considered in the context of the students, the subject and the course overall (see Hart, 1995:239). Students forming the basis of this study were completing a final year subject, Management Statistics, in the Bachelor of Social Science in Information Management at the University of Melbourne. In spite of the unfortunate title, the course was essentially a business degree with the majority of students having completed a year of professional practice prior to their final year and bringing considerable acumen and experience into the subject. In general, they were all quite keen students although somewhat hesitant at the thought of doing statistics as part of their course. Quite a number of students were enrolled part-time, having continued employment from their professional year, and found it difficult to juggle timetable requirements with their employment constraints.

For some years prior to the introduction of a web-based information system, Local Area Network-based systems were utilised to assist with the management of information flow between lecturer and students. There were 120 students enrolled initially in the course. Extensive use of AppleShare networks was made for students to collect course information, submit computer-based assignments and complete computer-based tests. 
The Macintosh network system was also used for students to collect and deliver weekly folio material for regular feedback, in the same way that normal tutorials do. Figure 1 shows the Statistics Icon on the LAN (Local Area Network) which was the central point for students to find relevant subject information.

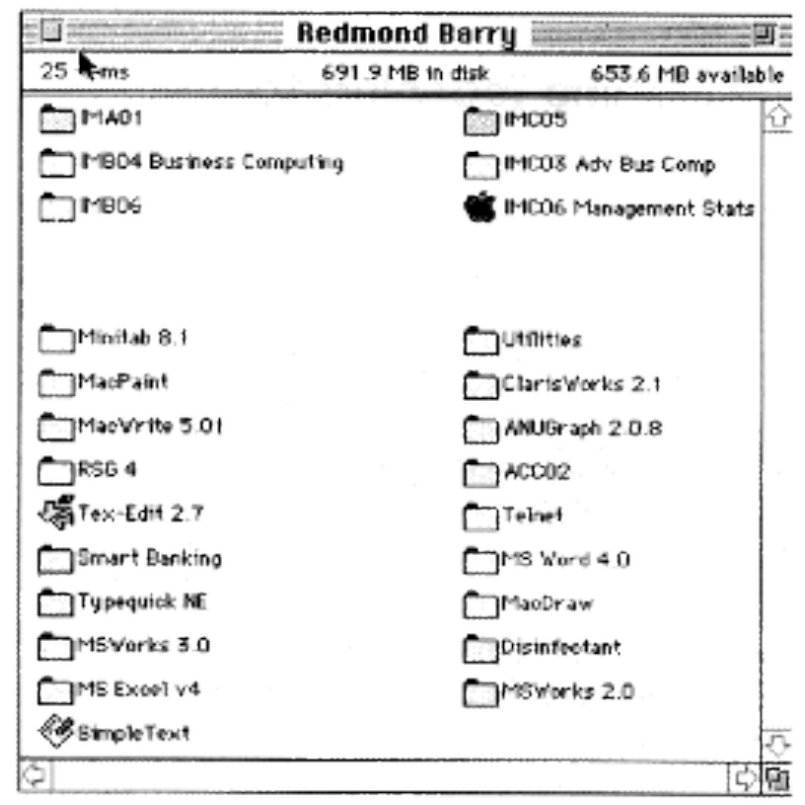

Figure 1: The Student LAN.

Once inside the Stats Icon, students could obtain information relevant to weekly activities or send information to their lecturer via a "drop folder" a one way process from student to lecturer. As can be seen in figure 2 below, folders were arranged to contain all files for each week with a separate folder for required readings and lecture demonstration files.

This system proved to be extremely robust and effective and resulted in considerable time savings with the collection and organisation of student work. One could say, in the Management of Information related to student progress. 


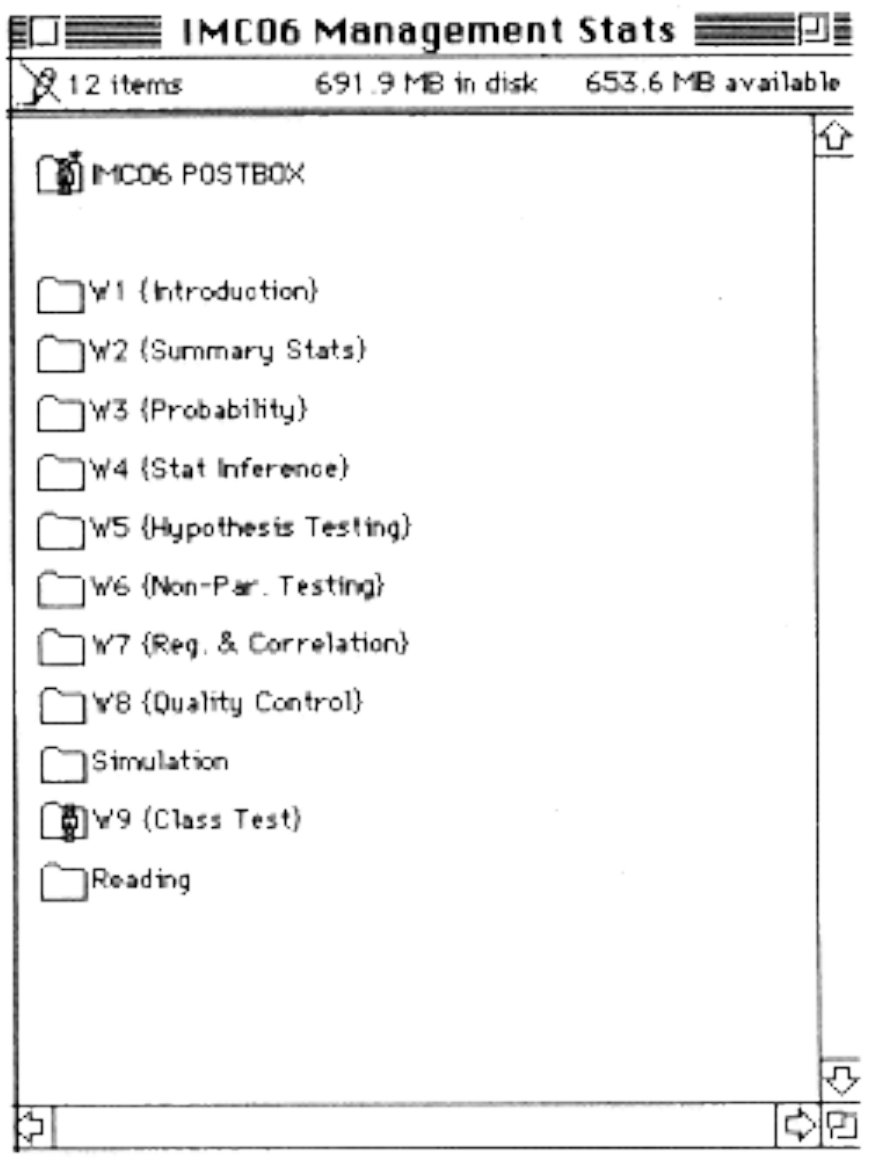

Figure 2: Showing folders inside the Statistics icon.

\section{So why use the web?}

When systems are working well, why change? With regard to implementing improvement in general to the quality of teaching, Paine, Turner and Pryke (1992:6) suggest: "It is very difficult to change when existing practices are comfortable, long established and appear to be successful." This is a moot point as any major change involves much work, the conversion from a LAN-based information system to a web-based system being no exception. However, while the Local Area Network operated extremely well using an AppleShare network, a problem existed in that the majority of students had IBM computers at home. Hence, there was often the requirement (not a great difficulty) to convert files from Apple Macintosh format to IBM format. The advent of the World Wide 
Web gave rise to the possibility of delivering course material on a truly platform independent medium. Information could be served up on a Macintosh and read on an IBM from any location. In this way, students simply required access to the Internet to access course material. Another drawback of the original LAN system was the lack of access for students from outside the university campus. Students required a UNIX account which was not available through this faculty. With increasing numbers of part time students, external access had become an important issue, particularly since the network was the single method available for students to submit their assessable work.

The web-based delivery of course material offered a very practical means of delivery of course material with the added benefit of communication through email. While it took some time to set the original system up, a great deal of time was saved in not being required to answer many student questions on 'trivia!' course matters as this information was centrally available. True, the early website offered no more than an electronic notice board and online course notes, but interestingly students appeared to actually read the material.

\section{The original website}

The course material that existed on the LAN was converted to HTML (Hypertext Markup Language) documents and a home page created to access information. Students were able to access the web site which was designed to be used as an online supplement to the usual physical teaching space. Students used the site as a central source of information on all aspects of the course, as well as to submit regular folio work. An email link on the web site allowed students to communicate with their lecturer, but as students did not have their own email account at this early stage, the communication was initially one way electronically with lecturer responses given collectively during following lectures and workshops or individually by printing out the student message. Over 400 individual student submissions were collected as part of the teaching feedback process during the semester. In 1996, student access to email has allowed significant innovations to be made.

A Power Macintosh 6100/60 was set up as a server using MacHTTP 2.0.1 software with standard protocols in the config file to serve up the website shown in figure 3.

The stats home page was modelled on the existing LAN system of placing relevant information in various "sections". A great feature of hypertext is that extensive links can be created to make relevant information readily accessible, and this was done to a certain degree in the modified documents. The main intention of this system, however, was not so much to improve the accessibility of information (this was already being done 
well via the LAN) but rather to improve communication from student to lecturer in the context of relevant activities. In this sense, the hypertext system worked extremely well with student feedback on weekly activities being automatically send to the lecturer's electronic mail account. This meant the lecturer didn't have to go and seek the information, rather the information came to him.

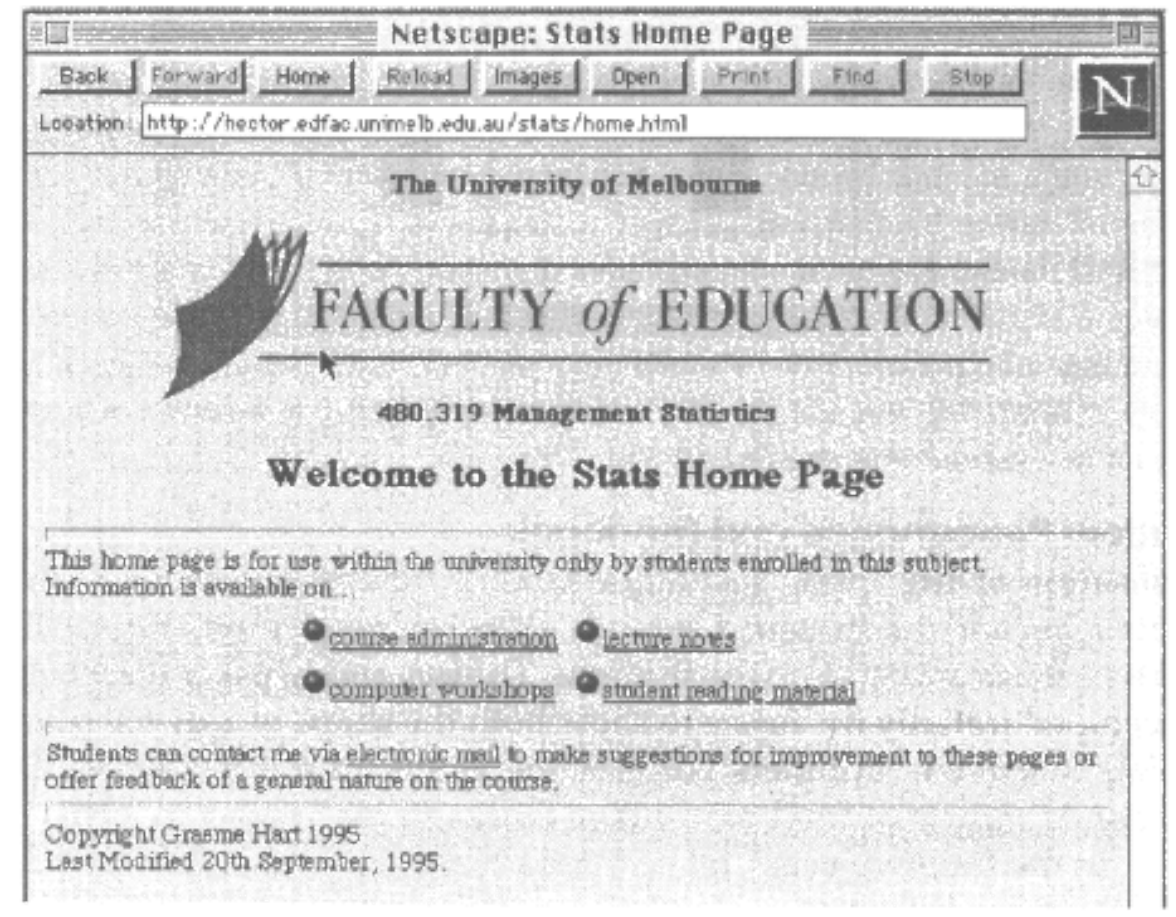

Figure 3: The Statistics home page on the World Wide Web.

Students still used the old LAN to send large completed Word files to a drop folder and to access examples of the Computer-Based-Test which they sat in their final workshop. This left the LAN with few files as shown in figure 4 .

The disadvantage of leaving files on the LAN was that the original problem of platform dependence still existed, albeit to a small extent, in that students were still required to access the AppleShare network to submit files for assessment. 


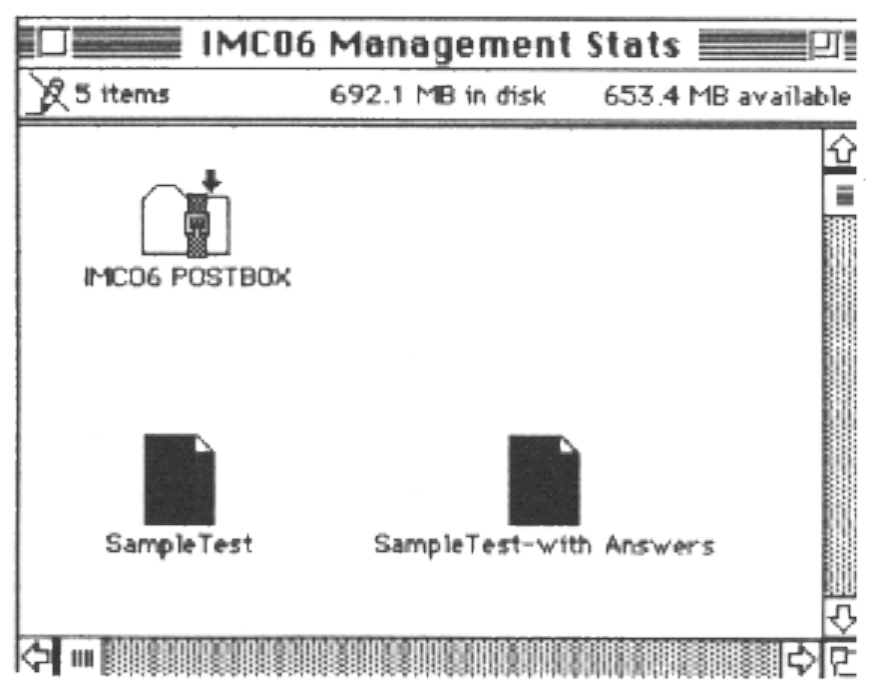

Figure 4: The "remains" of the original LAN.

\section{Student performance and feedback}

Utilisation of any "new" technique arouses interest and enthusiasm in both the teacher and the student. Certainly, if the teacher demonstrates a love of the subject, this transcends to the students. The box and whisker plots below were developed initially by Tukey to show both the shape of a distribution and to isolate so called "outliners" or extreme values in a distribution. The three diagrams represent final percentage marks in the subject in 1993, 1994 and 1995. In the first two years, a Local Area Network was used to distribute and collect course information. In 1995, a web based approach was used for the first time. It can be seen from the diagram that the median score in 1993 was $49 \%$, in 1994 it was 51\% and in 1995 the median score in the subject rose to $56 \%$.

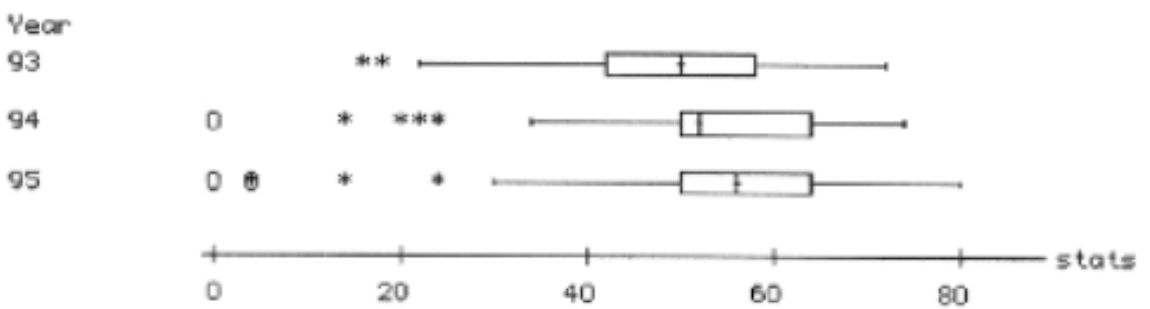

Figure 5: Box plots of student scores. 
While it is very tempting to believe that the introduction of a web based teaching system itself led to better "student performance", no such claim is made. However, there appears to be "something happening" here. The general novelty of increased access to course material, including readings and past papers, may well have been a factor in students performing better overall. While it is true that all the readings were available in previous years on short term loan from the library, the fact that they were so readily available from a central electronic source is a contributing factor according to student feedback.

Students in general were extremely positive about using the website. When asked what was "good" and what was "poor" about the site, there were very few students who commented on the negative aspects of using the web. While this was gratifying personally, it was disappointing professionally as students were encouraged and expected to offer constructive criticisms, given that they were headed for careers as professional Information Managers, to assist with the continual development of the site. Of the few who did make a criticism, their comments were along the lines of the student who said...

The only bad thing is that I couldn't get into the web at times, for instance, I have to check whether the lab is free. I still haven't had a chance to access the Web through other computer labs, perhaps I can get the web through other labs and the problem can be solved.

This comment is not about the site itself but to do with access to the laboratories. Of the comments about the "good" aspects of using the web, one student noted...

I have enjoyed using the WEB! I think that it is a quick and easy tool to use. The documentation is well done, it is nice to have all the information available to us without having to run around finding people to enquire [sic] about questions which have arisen.

A student who repeated the subject was in a useful position to be able to comment on the course attempted firstly without the web, then a second time with access to the web...

Having been fortunate enough to be involved in Stats both this year and last year I have found that the WEB facility available to us this year has made the course far superior to last year's course. Students are able to find all the relevant course information easily and quickly. There is little room for ambiguity for the student who is willing to look for information. The Stats Home Page was clearly set out and there was no confusion in the links to other documents. Overall I found having all the course information on the WEB that was easily accessible was fantastic. Thanks. 
It's interesting to note the "thanks" in the above comment. This was quite common from students and may be attributed to the novelty of using the web and an appreciation of being among the first to use this communications technology when it was clear that it would have a big role to play in future careers of students. This is supported by the student who suggested...

The web has been a very useful tool which has assisted me a great deal throughout the semester. It has cut the amount of time running around to find Lecturers to help solve problems. Nearly all information I have needed to know has been stored and has been extremely easy to access!!! It has been the best organising tool for the subject and I think other subjects and courses should take note of how it has been used in Stats. Well done Graeme for putting the effort and time to introduce Netscape to us and structuring your subject around it. No complaints at all from me. I will be looking out for the WEB in future employment as I am sure it will definitely impact every computer user around in a beneficial way.

In summary, the majority of the comments were positive about using the web because of there was a single central location to go to along with the convenience of being able to access information when it was required.

\section{The current website}

The current website, shown in figures 6 and 7, was designed after consideration of student feedback and an examination of the relative usage of various levels in the information hierarchy. Students were reluctant to go more that three or four layers down from the initial home page and so a website was designed with as much information as possible on the actual home page with most information being one level down from this. Very few links were more than two layers below the home page. This necessitated the use of a clickable map to present what was in fact a "course map" to assist navigation during the semester.

The page was designed with a view to the implementation of HTML frames in future designs. The administrative side of the course, with information on outline, assessment as so on, was placed down the left side and the course map completed the remainder of the page. A disadvantage of the clickable map was that is wasn't as easy to edit as an ordinary HTML document. In their article on designing virtual learning environments, Bergeron and Obeid (1995:140) claim that: "Clearly, for optimal educational value, users of computer based testing and learning 
environments must be given sufficient and appropriate clues as to how ... time can be expected to progress, and how their interactions will affect temporal progression." In a general sense, the progression of time over the semester was made clear with the "course map" approach detailing what was expected to be covered each week as the semester progressed.

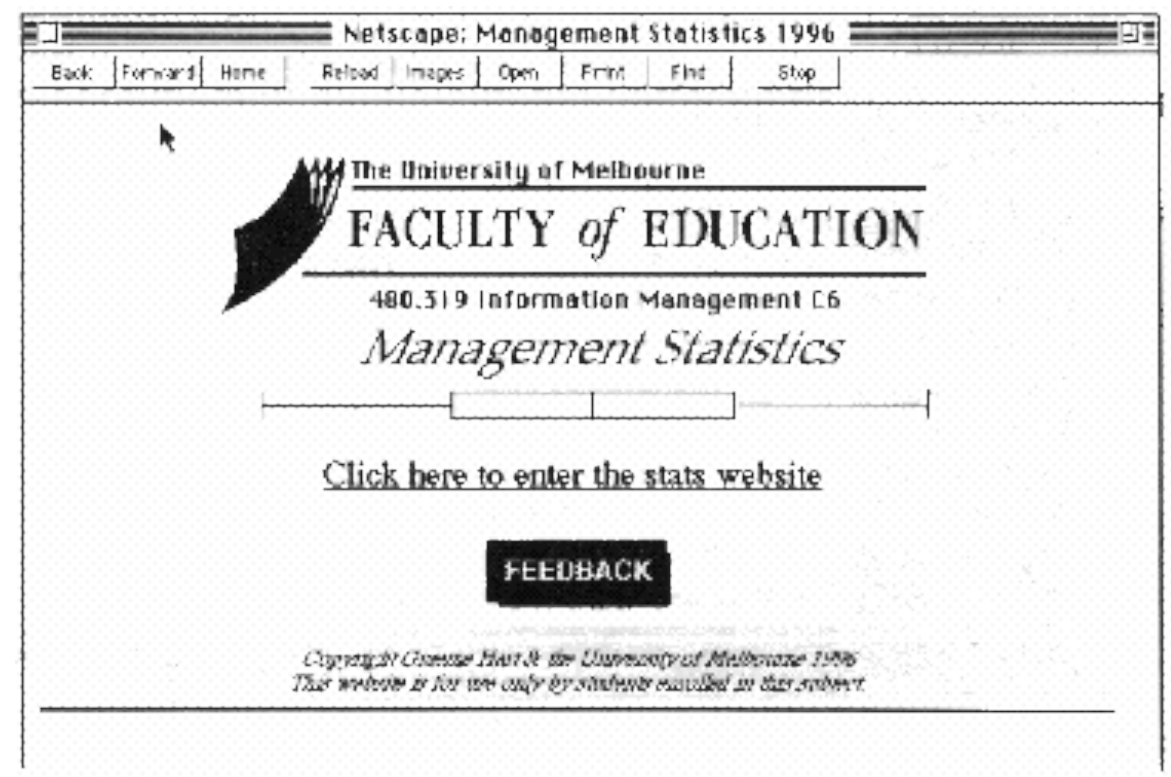

Figure 6: The opening screen.

Students were required to first access an opening screen, shown in figure 6 , with a link to the actual home page. This screen has two links, viz. a feedback link where students can send email to their lecturer without the need to activate their mail reader (Pegasus or Eudora) and a link to the actual home page. The site is currently open "to the world" but this approach gives the option of requiring a username and password to access the actual teaching space. This feature will be employed next year to enable tracking of student usage as part of ongoing research into the relationship between student learning styles and use of hypertext in web documents (see Hart, 1995). 


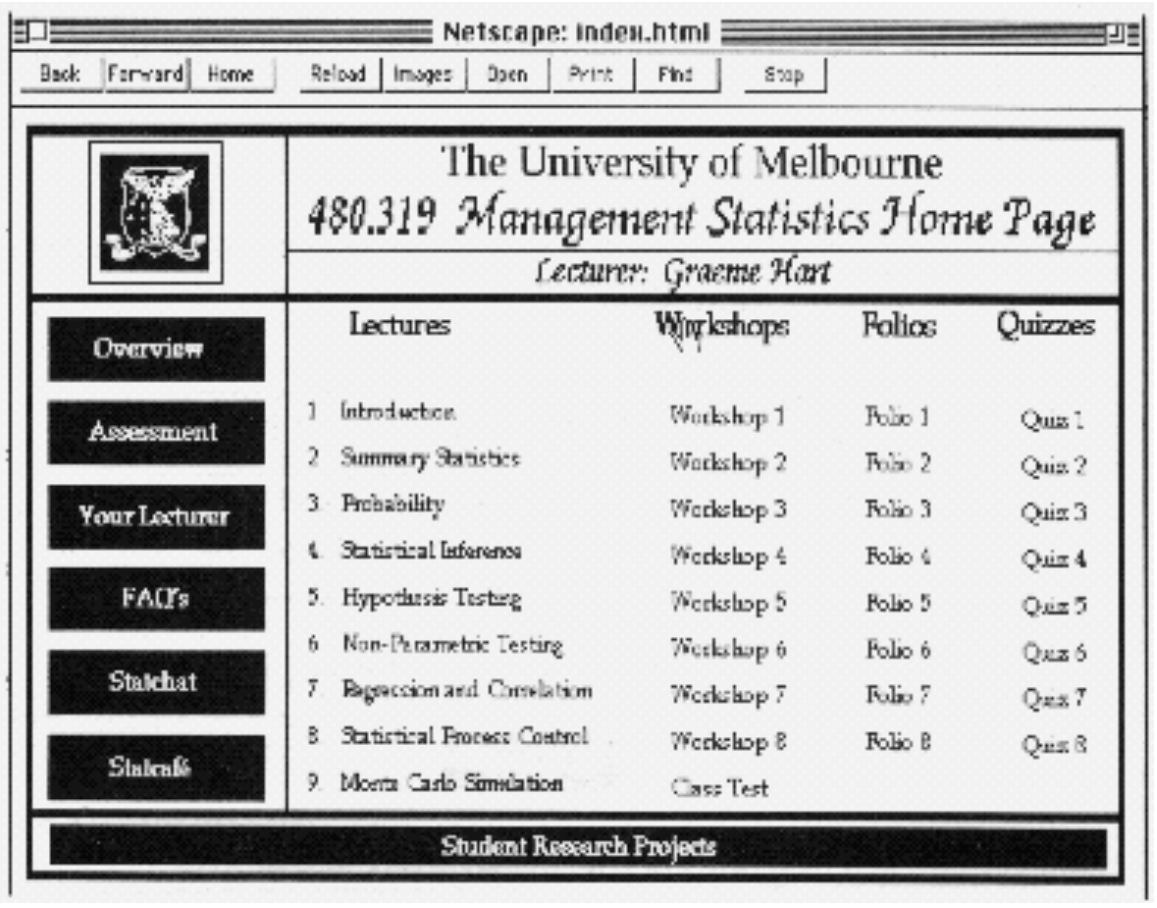

Figure 7: The current Stats home page.

In a typical teaching environment, teachers need to be able to give information to students in the first instance, gather feedback from students and stimulate interaction and discussion to begin the teaching process. The home page reflects this approach with sections divided into:

- Course information

- Course materials and activities

- Communication with lecturer

- Contributions from students

\section{Course information}

The course information consists of the usual overview and assessment information which was distributed in paper form at the beginning of semester. This was a requirement for all subjects. Any information 
available as a hardcopy handout was shown with a white background. In that way, students knew if printed copies of larger documents were available. It was found with the previous website that many students preferred to print out hardcopies of certain documents and this was done in advance for certain lecture notes, and all of the workshop activities.

\section{Course materials and activities}

This was the working area of the website where a "course map" was provided to assist student navigation and to reduce the possibility of getting "lost in cyberspace". The map approach worked extremely well and students had no problems in finding information relevant to activities for a particular week. The subject was delivered in the standard way with two hour lectures giving the theory with appropriate demonstration solutions, the corresponding two hour workshops covering the solution of problems related to the theory both by hand in the first hour then using Minitab software during the second hour, with assessable folio activities designed to draw together the content of both the lectures and workshops which students completed in their own time. Lectures, given their nature, were essentially a one way process of lecturer to student; the workshops, as far as was possible, were an interactive time with discussion between tutor and student as well as student and student. The folio activities were a one way process from student to lecturer with the completed submission being handled by a Common Gateway Interface (CGI) script and sent to a remote file. This differed from other email interaction as all student submissions were "stacked" into the one file with the date and time of submission attached and so all student folios could be read in the one "sitting" and feedback given during lectures in the following week.

The quizzes consisted of ten or so multiple choice questions designed to give students the opportunity to get a small amount of instant feedback on their general understanding of the topic each week. The quizzes were not interactive but are currently being developed to use Shockwave technology for a high level of on screen interactivity and feedback in next year's course.

Colour coding was used in that all quizzes had a yellow background, the folios were green and the workshops, because they were available in a hardcopy "booklet", were white. Colouring the backgrounds appeared to have little impact on helping students navigate as they found the system easy to use anyway, but they were of assistance during the practical computer workshops in gaining an overview of where students were "at". One student had the temerity to be playing a game during one workshops and she was easily singled out with a different coloured screen! 


\section{Communication with lecturer}

The use of online systems compared with traditional methods clearly have a great advantage in the distribution of course information. This comes through time and again in student feedback. But this is only part of the advantage. If online systems are to make a contribution to the teaching and learning process beyond simple convenience, they must improve the communication between teacher and student. Communication is the key to the teaching-learning process. The link on the home page to "Your Lecturer" gave students information on the usual phone and fax numbers as well as times of availability and an email link for electronic communication. A major limitation of the web system used in 1995 was that of Lecturer-Student communication. In essence, because of the limitations with lack of student access to email, the process was electronically one way as represented in figure 8 .

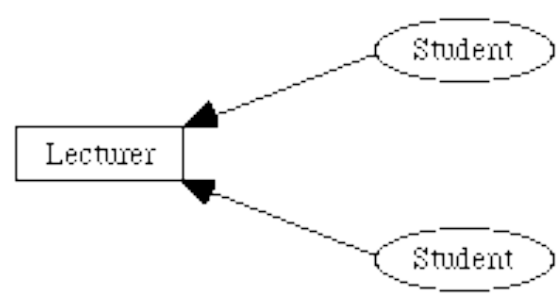

Figure 8: Limitations of LAN based communications.

The advent of student access to their own electronic mail account has allowed continual development in the attempt to replicate the traditional teaching situation in a classroom by virtual means. Students were able to have both individual responses from their lecturer and be part of a global chat via a listserver known euphemistically as "STATCHAT". This was intended to create a virtual "teaching space" along the lines shown in figure 9 .

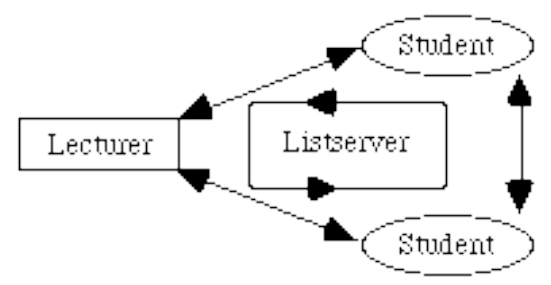

Figure 9: Lines of electronic communication.

In 1995, many students, particularly those enrolled part time, expressed the desire to complete their work in distance mode. In 1996, students were 
offered the opportunity to enrol in "virtual workshops" where communication was predominantly by electronic mail. Approximately one quarter of the students chose this option: research on the success or otherwise of this feature in progress and not the subject of this paper.

\section{Contributions from students}

Besides the formal feedback mechanism of the folio activities and the use of electronic mail, an interesting event occurred during the latter part of the semester. It was explained to students that their research projects, making $30 \%$ of their overall assessment and submitted electronically as a wordprocessing document, were to be converted to Adobe Acrobat documents and served up on the website in the "Student Research Projects" link. In this way the students would be able to "publish" their research for others to see. Incidentally, besides being intended to excite the students as a novel way of presenting reports, this was intended as a small "experiment" to stop plagiarism, often the scourge of electronic based subjects.

A small group of students became interested enough in the notion of having their work on the web that they requested their own space on the home page. This was duly created as the "Statcafe" link which was effectively a location students could write files to using File Transfer Protocol (see Liu et al, 1994). In this way, documents such as suggested solutions to problems could be shared between students on a collective basis. Because the student space was created later in the semester, it did not gain a great following, but the concept will be expanded upon in the next website. Empowering the students to control their own part of the online teaching space had a marked positive effect on those who were involved, a view supported by Awbrey (1996:17) in her assertion that: "Emerging technologies can free the instructor to act as a catalyst of student inquiry and dialogue, creating environments as never before that fulfil John Dewey's vision of active, engaged learners."

\section{Discussion}

There is little doubt that online systems, particularly those based on the World Wide Web, will become more common in the near future. There appear to be two camps of interest, one interested in the pedagogy of the technology and the other interested in the pragmatics. Most of the research into Internet based systems in education focuses on the impact on the teaching-learning process. (see Eckuld, et al 1995) while much of the push from higher levels appears to be based on the rationale of attracting more students. Given the comments by the Victorian Auditor General that The University of Melbourne "... should note trends in 'distance learning' programs such as the open learning programs and the Internet, which could have a 'potentially dramatic' impact on the university's space needs" 
(Martin, 1996), it's not surprising that senior education management are taking an interest.

Universities in particular, and educational institutions in general, are becoming more "business oriented" and adopting strategies to attract the best students, with Internet offerings seen as part of the package. The push for quality measures in higher education is also part of the overall change towards the offering of a product or service and the willingness to adapt to changes in the marketplace. Many would argue against this trend but as Davidow and Malone (1992:16) argue: "Whether it is producing a product or a service ... the virtual corporation alone has the speed and flexibility to cope with ... a pace of change that will make contemporary business life seem glacial.' As universities become more oriented to online delivery of their services, they also become more flexible in coping with change. Perhaps this is best summed up as:

The next decade and century will see increasingly intensive use of various modes of distance education.... The reasons for such an increase are clear: There are new technologies seeking new markets, important political agendas, especially at the international level, sufficient novelty to intrigue anyone, empires to be built, and compelling needs for access justifiable on moral grounds (Holloway and Ohler, 1991:259).

This paper has outlined the development of a web based approach to teaching a statistics subject at The University of Melbourne. The subject lent itself to an orderly, regulated and somewhat linear approach which may not be appropriate to other disciplines. In this particular situation the provision of course material on the web was extremely effective and well received by students. The management aspects of teaching with the delivery and collection of information and student submissions worked seamlessly and well. Whether the system operated effectively in improving the actual communication between lecturer and students is debateable. This paper has been prepared before the end of semester and before a thorough analysis of student comments, web usage and results can be completed, but anecdotal evidence suggests that while a certain proportion of students engaged in electronic communications with a passion, others appeared to be somewhat disenfranchised by the technology and rarely communicated electronically. Thus far, there have been around 600 email communications between lecturer-student and student-lecturer seemingly along the lines of the 80-20 Pareto Principle of $20 \%$ of the students providing $80 \%$ of the communication. This is probably no different when compared with the traditional teaching environment will be part of a future research paper. The same appears to be true of the faculty staff. Hart and Andrew (1996) are currently researching staff attitudes to the introduction of online teaching methods with technological troglodytes and enthusiasts balancing the equation. No new teaching programs can succeed unless staff support them with vigour with the 
computer literacy of teachers (see Mason and Fisher, 1995) a tremendously important aspect of any program to deliver courses online.

In a short time, the suggestions for a model online teaching space made in this paper will appear antiquated. This is certainly a desired outcome since many options exist for new and improved technological ways to deliver educational programs are already with us. The convenience of online delivery in the management aspect of education is apparent for both teacher and student; whether the actual learning process is enhanced at this stage is debateable and warrants further investigation.

\section{References}

Awbrey, S. (1996). Successfully integrating new technologies into the higher education curriculum. Educational Technology Review, Winter 1996, No. 5.

Bergeron, B. and Obeid, J. (1995). Temporal issues in the design of virtual learning environments. Journal of Educational Multimedia and Hypermedia, 4(2/3).

Davidow, W. and Malone, M. (1992). The Virtual Corporation: Structuring and Revitalising the Corporation for the 21st Century. Harper Collins, 1992.

Eckuld, J., Garrett, P., Ryan, S. and Harvey, J. (1995). Designing the world wide web as an educational delivery medium at the University of Sydney. In Pearce, J. and Ellis, A. (eds.), Learning With Technology, 132-138. Science Multimedia Teaching Unit, The University of Melbourne.

Hart, G. (1995). Learning styles and hypertext: Exploring user attitudes. In Pearce, J. and Ellis, A. (eds.), Learning With Technology, 238-245. Science Multimedia Teaching Unit, The University of Melbourne.

Hart, G. and Andrew, P. (1996). Impact of the introduction of web based teaching on academic staff. In WebNet96 Proceedings, Association for the Advancement of Computing in Education (yet to be published).

Holloway, R. and Ohler, J. (1991). Distance education in the next decade. In Anglin, G. (ed.), Instructional Technology: Past, Present and Future, 259-266.

Liu, C. Peek, J., Jones, R.. Buus, B. and Nye, A. (1994). Managing Internet Information Services. O'Reilly and Associates.

Martin, L. (1996).Uni told to manage its properties better. The Melbourne Age, 31 May 1996, page A4.

Mason, J. and Fisher, A. (1995). Origins and development of computer literacy for the 90s: An in-service for teachers. In Pearce, J. and Ellis, A. (eds.), Learning With Technology, 376-379. Science Multimedia Teaching Unit, The University of Melbourne.

Paine, J., Turner, P. and Pryke, R. (1992). Total Quality in Education. Ashton Scholastic.

Graeme Hart is in the Faculty of Education at the University of Melbourne, Parkville Vic 3052, Australia. Email g.hart@edfac.unimelb.edu.au. http:/ / hector.edfac.unimelb.edu.au/

Please cite as: Hart, G. (1996). Creating an online teaching space. Australian Journal of Educational Technology, 12(2), 79-93. http: / / www.ascilite.org.au / ajet/ajet12 / hart.html 